\title{
Addendum: Activating lattice oxygen redox reactions in metal oxides to catalyse oxygen evolution
}

Alexis Grimaud, Oscar Diaz-Morales, Binghong Han, Wesley T. Hong, Yueh-Lin Lee, Livia Giordano, Kelsey A. Stoerzinger, Marc T. M. Koper and Yang Shao-Horn

Nature Chemistry 9, 457-465 (2017); published online 09 January 2017; addendum published after print 23 June 2017.

In our Article we reported direct experimental evidence for the involvement of lattice oxygen redox chemistry in the perovskitecatalysed oxygen evolution reaction (OER). We would like to cite an Article ${ }^{1}$ that was published prior to ours that readers should be aware of. The Article reports the OER activities of a series of cobaltite perovskites $\left(\mathrm{La}_{1-x} \mathrm{Sr}_{x} \mathrm{CoO}_{3-\delta}\right)$, and its authors rationalize the high activities for materials with $x>0.4$ through the participation of lattice oxygen in the OER mechanism, a hypothesis that is supported by density functional theory.

\section{References}

1. Mefford, J. T. et al. Water electrolysis on $\mathrm{La}_{1-x} \mathrm{Sr}_{x} \mathrm{CoO}_{3-\delta}$ perovskite electrocatalysts. Nat. Commun. 7, 11053 (2016). 